\title{
ENGINEERING MICROSCALE BIOMIMETIC HYDROSCAFFOLD FOR DYNAMIC THREE-DIMENSIONAL MODELING OF PANCREATIC CANCER
}

\author{
Frédérick de Miollis ${ }^{1,2,4}$, Zied Souguir ${ }^{3}$, Joseph de Saxcé ${ }^{4}$, Lucie Dercourt ${ }^{1}$, Elodie Vandenhaute ${ }^{3}$, \\ Nathalie Maubon ${ }^{3}$, Isabelle Van Seuningen ${ }^{2 *}$, Vincent Senez ${ }^{1 *}$ \\ ${ }^{1}$ CNRS, Univ. Tokyo, UMI 2820 - LIMMS, Lille F-59000, France \\ ${ }^{2}$ Univ. Lille, CNRS, Inserm, CHU Lille, UMR 9020-U 1277 - CANTHER, Lille F-59000, France \\ ${ }^{3}$ HCS-Pharma, Loos F-59120, France \\ ${ }^{4}$ Univ. Lille, CNRS, UMR 8520 - IEMN, Villeneuve d'Ascq F-59650, France \\ ${ }^{*}$ Both authors contributed equally to the work
}

\begin{abstract}
Pancreatic cancer (PC) is a deadly cancer for which no diagnostic or prevention plan currently exists since this cancer is asymptomatic in its early development. The very specific and complex tumor microenvironment of PC is responsible for the unsuccessful delivery of therapeutics molecules to tumor cells. For high throughput testing of new molecules, there is a crucial need for more accurate preclinical model. For the first time, we integrated in a microfluidic system 5 major features of PC microenvironment: i) type-I collagen (CI), ii) hyaluronic acid (HA), iii) pancreatic stellate cells (SC), iv) interstitial fluid flow and v) nutrient and oxygen gradients. We showed long 3D culture (30 days). Furthermore, we implemented, thanks to the perfusion capability, the actual FOLFIRINOX protocol to assess the IC50 and we showed that dynamic conditions induce increased chemoresistance compared to 3D static.
\end{abstract}

\section{KEYWORDS}

Pancreatic cancer, organ-on-a-chip, hyaluronan hydroscaffold, FOLFIRINOX, tumor microenvironment, BIOMIMESYS.

\section{INTRODUCTION}

Announcing PC is always synonym of death for the patient. Its incidence equals its mortality. Treatment whenever possible (10-15\% of cases) implies a heavy and complex surgery. For the rest of the patients (80-85\%) diagnosed with late and metastatic cancer, only palliative chemotherapeutic treatment is offered. These treatments (gemcitabine, FOLFIRINOX) have low efficacy (several months survival median) and show high toxicity, often due to multifactorial resistance [1].

One of the major reasons of chemotherapeutic treatment failure in PC is the limited or inefficient delivery of drugs to tumor cells (TC). Indeed, PC is one of the most stromal-rich cancers. In many cases, only $10-40 \%$ of the pancreas tumor is composed of TC, while the remainder is composed of pancreatic stellate cells (SC), fibroblasts cells (FC), myofibroblasts cells , immune cells , blood vessels, extracellular matrix (ECM), cytokines and growth factors [2]. This dense and bulky microenvironment prevents normal vascularization of the tumor amplifying the inefficient pharmaco-delivery. Indeed, CI induces higher solid stress [3], HA prevents drug delivery [4], SC are key players in PC [5], interstitial fluid modifies drug distribution and protein expressions, finally hypoxia in PC microenvironment promotes tumor survival [6].

Most studies have been limited to in vitro 2D \& 3D static models [7] or in vivo approaches using xenograft tumors in immunodeficient murine models [8]. Few microfluidic PC models have been described in the literature. Drifka and coworkers patterned TC with either FC or SC in hydrogels containing both CI and HA [9]. However, culture time was limited to 3 days. Beer and coworkers worked on PC spheroids integrated in a microfluidic system and showed increased chemoresistance against cisplatin compared to static conditions [10]. Gioeli and coworkers dynamically cocultured TC, SC and endothelial cells in CI [11]. However, they did not integrate HA and did not control oxygen and glucose distribution. Finally, Lee and coworkers implemented a TC and SC co-culture in CI without HA [12]. To our best of knowledge, no work has been reported on implementation of actual FOLFIRINOX protocol in 3D dynamic model of PC.

\section{MICROFLUUIDIC SYSTEM}
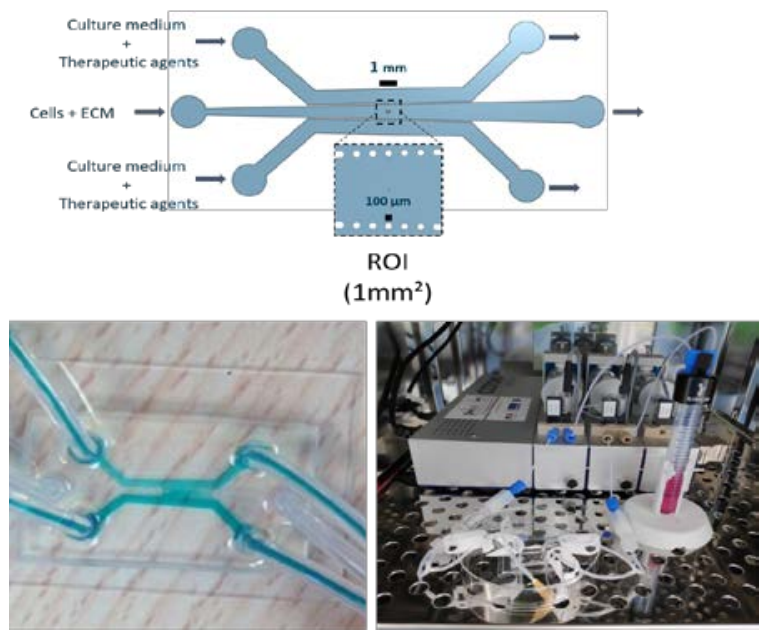

Figure 2: (top) Schematic of the microfluidic system and description of its implementation as a $3 D$ dynamic culture reactor for PC model; (bottom-left) Actual device made of PDMS \& glass and dynamically perfused with blue colored water; (bottom-right) Photo of the device connected to the syringe pump and culture medium reservoir in the incubation chamber. 
The microfluidic device is composed of a $1.0 \mathrm{~mm}$ wide central gel-chamber, with rounded loading reservoirs at both ends for hydrogel + cell loading, connected through 5 hexagonal pillars $(100 \mu \mathrm{m}$ diameter) regularly spaced (gap=100 $\mu \mathrm{m}$ ) to two side medium-chambers (1mm wide) end-closed with rounded loading reservoirs and designed to bring nutrient, oxygen as well as drugs to the tumor model in the central chamber (Figure 1). The microfluidic device was fabricated using standard polydimethylsiloxane (PDMS) soft lithography. The geometry of the device is inspired by the works of Cho [13] and Huang [14]. The key step in the fabrication process is the filling of the hydrogel in the central channel without spreading in the side channel. The pinning of the gel interface between posts depend on the spacing between posts, the wetting properties of the hydrogel versus polydimethylsiloxane/glass, width of the central channel and viscosity of the hydrogel. During gel injection, a pressure difference (modeled by the YoungLaplace (Y-L) equation) is created at the gel/air interfaces (Figure 2).

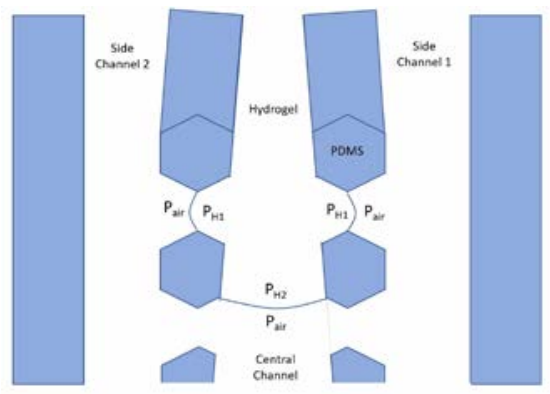

Figure 2: Schematic of a filling process in the central channel. During injection, a difference in pressure occurs at the air-liquid interfaces $\left(P_{\mathrm{H} 1}-P_{\text {air }} \& P_{\mathrm{H} 2}-P_{\text {air }}\right)$ and robust filling is achieved when $P_{\mathrm{H} 2}-P_{\text {air }}$ is above local $Y-L$ conditions (advancing interface) while $P_{H 1}-P_{\text {air }}$ is below $Y$ $L$ criterium (pinned interface). Minimizing the hydrodynamic resistance of the channel by enlarging the section toward outlet allows $100 \%$ fabrication yield.

The pressures on the gel side $\left(\mathrm{P}_{\mathrm{H} 1} \& \mathrm{P}_{\mathrm{H} 2}\right)$ depends on the hydrodynamic resistance of the path between the inlet of the device and the location of the interface. If these pressures exceed the Y-L conditions, the interface moves forward and the gel fills the empty space. Since the pressure in the gel decreases along the channel, bursting of the gel in the side channel, if it happens, necessarily occurs at the level of the first gap. It means that the pressure in the gel at the level of the interface between the two first posts $\left(\mathrm{P}_{\mathrm{H} 1}\right)$ have to be low enough to fulfill the $\mathrm{Y}-\mathrm{L}$ local conditions and so prevent bursting while at the level of the interface between the central channel wall $\left(\mathrm{P}_{\mathrm{H} 2}\right)$, it should be high enough to induce interface displacement and channel filling. It follows that the hydrodynamic resistance of the channel has to be lowered in order to minimize the pressure drop along the channel. We have numerically (COMSOL MULTIPHYSICS) studied the filling process of various channel geometries: a) narrowing, b) venturi, c) constant, d) inverse venturi and e) enlarging (Figure 3). We used the 'microfluidics' module to solve the transient filling of the central channel and defined the optimal geometry preventing the hydroscaffold spreading in the side channels. We found that the 'enlarging' geometry (widths: at inlet $=500 \mu \mathrm{m}$, at outlet $=1200 \mu \mathrm{m}$, channel length $=21000 \mu \mathrm{m}$ ) provides the best conditions to yield a $100 \%$ success in the filling process with a pressure drop between inlet/outlet of $1500 \mathrm{~Pa}$.
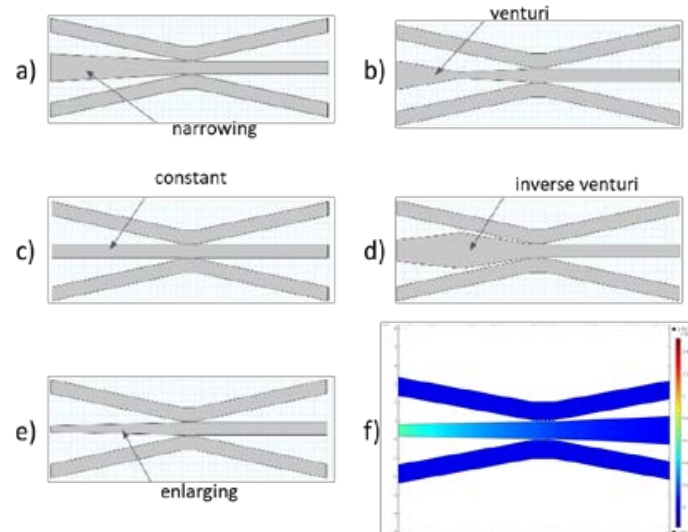

Figure 3: Schematic description of the different geometries modeled with COMSOL MULTIPHYSICS in order to make robust the filling of the central channel with hydrogel.

\section{D MICROFLUIDIC PC MODEL \\ BIOMIMESYS (HA hydroscaffold) synthesis and use}

To better mimic the tumor microenvironment, HCS Pharma develops BIOMIMESYS ${ }^{\circledR}$ Oncology, a double interpenetrating network (IPN) hydroscaffold based on Hyaluronic Acid (HA-1.6 MDa) and type I collagen crosslinked with Adipic acid dihydrazid (ADH). Its Young modulus is $1.0 \pm 0.2 \mathrm{kPa}$ and its porosity is $155 \pm 35 \mu \mathrm{m}$. For 3D static culture, BIOMIMESYS ${ }^{\circ}$ Oncology is provided as 3D lyophilized sterile hydrogel domes in multi-wells format. For 3D dynamic conditions, we used tube clamp to close lateral channel and we injected BIOMIMESYS ${ }^{\circledR}$ Oncology in the central channel with a needle syringe. The hydroscaffold is then carried out using HCS Pharma process and stored at $4{ }^{\circ} \mathrm{C}$ in Sterilsop SX Sterilization sheaths.

\section{Mass transport in BIOMIMESYS hydroscaffold}

Dynamic stresses in living tissues drive interstitial flows through ECM that transport oxygen/nutrients/therapeutic molecules throughout the tissue and have important role in chemoresistance [15].

Using COMSOL MULTIPHYSICS (COMSOL FRANCE, Grenoble, France) and the 'reactive flow in porous material' module, we modeled the 3D distribution of oxygen in the perfused microdevice taking into account both diffusion and advection. We approximate the porous properties of the central channel filled with the HA-C1 hydro-scaffold and TC+SC with a constant porosity of 0.994 and a permeability of $5.010^{-12} \mathrm{~m}^{2}$. The diffusive transport of oxygen was modeled with a diffusion coefficient of $2.6910^{-9} \mathrm{~m}^{2} / \mathrm{s}$ with a Bruggeman correction to include tortuosity effect in the porous media [16]. Oxygen boundary concentration was fixed by the operating conditions in the incubator $\left(0.174 \mathrm{~mol} / \mathrm{m}^{3}\right)$. The purpose was to tune the syringe flow rate to obtain an average velocity of interstitial flow closed to its in vivo value ( $\sim 5$ $\mu \mathrm{m} / \mathrm{s}$ [17]). The simulation showed that a syringe flow rate 
of $10 \mathrm{nl} / \mathrm{s}$ ensure the right conditions and induced oxygen gradient in the central channel.

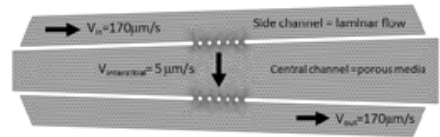

a) geometry

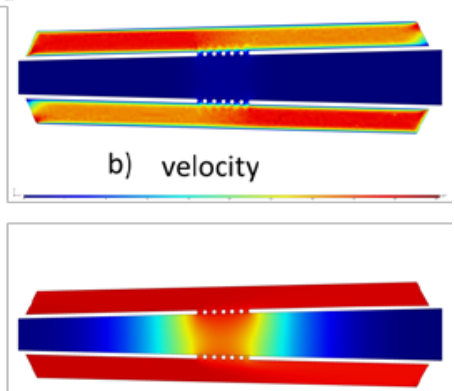

c) oxygen

Figure 4: a) $X-Y$ view of the $3 D$ geometry of the microfluidic device with targeted average flow velocities to induce in vivo like interstitial fluid advection; b) resulting culture medium velocity distribution; c) corresponding oxygen distribution.

We tested the fluid transport in the porous BIOMIMESYS scaffold placed in the central channel using fluorescein (SIGMA - M.W. 332 Da). The central channel was filled with collagen $1(2 \mathrm{mg} / \mathrm{ml})$ without cells and the lateral channels with DPBS $1 \mathrm{x}$. One of the lateral channel is perfused with fluorescein $(1 \mathrm{mg} / \mathrm{ml})$ in DPBS $1 \mathrm{x}$ at a flow rate of $10 \mathrm{nl} / \mathrm{s}$. Images are taken every 5 seconds with DMi8 inversed microscope (LEICA) with CMOS Orca Flash 4 camera (Hamamatsu, Tokyo, Japan) associated to LAS X software. Time (t) imaging module was used. Images were then analyzed with Fiji software by ImageJ. Frame per frame analysis was performed to calculate the displacement of the fluorescein front line. (Fig. 5). The measured velocity perpendicular to the direction of the central channel is found to be 3,493 $\mu \mathrm{m} / \mathrm{s}+/-0,152 \mu \mathrm{m} / \mathrm{s}$ in agreement with modeling.
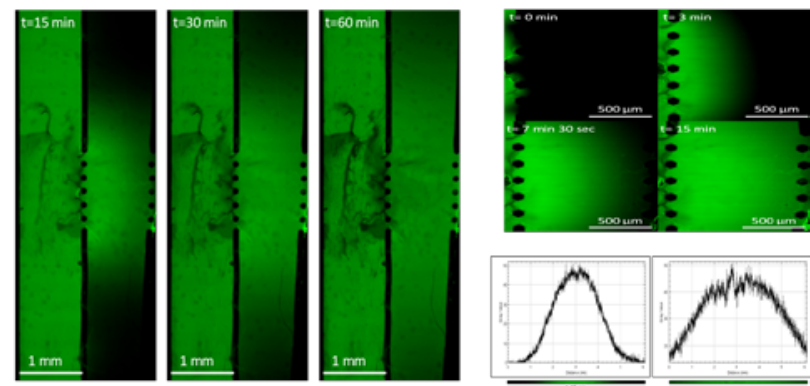

Figure 5: (left) Photos of the top view of the microfluidic device showing the displacement of the fluorescein front at 15, 30 and 60 minutes; (right-top) zoomed picture of the fluorescein transport between 0 and 15 minutes; (rightbottom) $1 D$ profile of the fluorescein distribution along the central channel at 15 \& 60 min.

\section{Stock cell culture}

SC were isolated from clinically diagnosed human pancreas (UK Human TissueBank, Trent MREC, 05/MRE04/82) and immortalized with retrovirus [18]. SC were maintained in DMEM/F-12 + 10\% (v/v) FBS + 1\% $(\mathrm{v} / \mathrm{v})$ Pen Strep $+1 \mu \mathrm{g} / \mathrm{ml}$ Puromycine media. Commercially available TC (Capan-2, ATCC ${ }^{\circledR}$ HTB$80^{\mathrm{TM}}$ ) expressing epithelial phenotype were routinely maintained in media (McCoy's 5A Medium $+10 \%(\mathrm{v} / \mathrm{v})$ FBS $+1 \%$ (v/v) Pen Strep $+1 \%(v / v)$ L-glutamine) at $37{ }^{\circ} \mathrm{C}$ in an incubator containing $5 \% \mathrm{CO}_{2}$ and either passaged or used once a week culture.

\section{Three-dimensional cell co-culture}

We prepared $1 \mathrm{ml}$ of cells laden collagen 1 solution

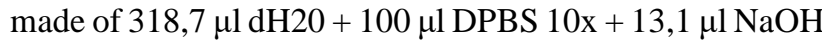
$+568,2 \mu \mathrm{l}$ collagen $(2 \mathrm{mg} / \mathrm{ml}$ ) (Corning) and kept it on ice before injection. We added 2 million cells $/ \mathrm{ml}$ with a ratio SC:TC of 3:1 in the $1 \mathrm{ml}$ CI solution. For 3D static culture, we deposited $20 \mu \mathrm{l}$ of the solution on the BIOMIMESYS dome and incubated for $1 \mathrm{~h}$ at $37^{\circ} \mathrm{C}$. Then, we added the complete culture medium and incubated at $37^{\circ} \mathrm{C}$. For $3 \mathrm{D}$ dynamic culture, we closed the side channel with tube clamps, homogenized the CI solution with cells, and injected $50 \mu \mathrm{l}$ in the central channel. We closed the central channel and incubated $1 \mathrm{~h}$ at $37^{\circ} \mathrm{C}$. We connected the device to the neMESYS syringe pumps (CETONI GmbH, Korbußen, Germany) and perfused culture medium at 10 $\mathrm{nl} / \mathrm{s}$ and $37^{\circ} \mathrm{C}$. Culture time was set to 7 days before analyses but we can easily reach more than 30 days.

\section{Activation of SC}

SC are one of the most important stromal cells in the microenvironment of PC. Their persistent activation creates conditions favorable for PC [19]. Rigidity properties of ECM modulate their activation. We compared the effect of Matrigel and our HA/CI matrix on the activation of SC in 3D static culture conditions. Live/Dead tests was used to attest that SC are alive (Fig. 6). We found SC stayed quiescent in Matrigel while they are activated (star shape) in our new ECM model.

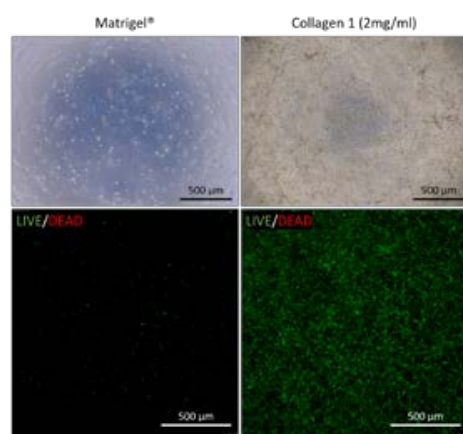

Figure 6: (Left) 3D culture of SC in Matrigel; (Right) 3D culture of SC in collagen $1(2 \mathrm{mg} / \mathrm{ml})$. SC are quiescent in Matrigel (round shape) meanwhile in collagen they form a network and have their star-like shape.

Live/Dead test (Invitrogen, Carlsbad, CA) was used stains dead cells with red-fluorescent ethidium homodimer-1 (EthD-1) and live cells with green fluorescent calcein-AM. The culture medium was removed and $200 \mu \mathrm{l}$ of $2 \mu \mathrm{M}$ calcein-AM and $4 \mu \mathrm{M}$ EthD- 1 mixed in DPBS 1x solution was added to each well and incubated 
at room temperature for 30 minutes. Imaging was performed by confocal spinning disk microscopy (AxioObserver Z1, Zeiss, Marly le Roi, France).

\section{Device operation for drug screening}

FOLFIRINOX is a standard treatment for patients with PC but has efficacy rates of less than 32\% [20]. We prepared the 3 chemotherapeutic stock solutions in Dimethyl Sulfoxide (DMSO). These 3 drugs were then mixed to culture medium and added at different concentrations (Table 1 ) to the 3D static/dynamic culture at $37^{\circ} \mathrm{C}$ for $72 \mathrm{~h}$.

Table 1: Concentration of the different chemotherapeutic molecules of the FOLFIRINOX treatment used for the IC50 assessment.

\begin{tabular}{ccccccccc}
\hline & Dose 1 & Dose 2 & Dose 3 & Dose 4 & Dose 5 & Dose 6 & Dose 7 & Dose 8 \\
\hline 5.Fu & 0 & $4 \mathrm{nM}$ & $40 \mathrm{nM}$ & $0,4 \mu \mathrm{M}$ & $4 \mu \mathrm{M}$ & $40 \mu \mathrm{M}$ & $400 \mu \mathrm{M}$ & $4 \mathrm{mM}$ \\
oXA & 0 & $0,5 \mathrm{nM}$ & $5 \mathrm{nM}$ & $50 \mathrm{nM}$ & $0,5 \mu \mathrm{M}$ & $5 \mu \mathrm{M}$ & $50 \mu \mathrm{M}$ & $500 \mu \mathrm{M}$ \\
SN-38 & 0 & $12,5 \mathrm{pM}$ & $125 \mathrm{pM}$ & $1,25 \mathrm{nM}$ & $12,5 \mathrm{nM}$ & $125 \mathrm{nM}$ & $1,25 \mu \mathrm{M}$ & $12,5 \mu \mathrm{M}$ \\
\hline
\end{tabular}

For the 3D dynamic culture conditions, we first injected the mixture of the 3 drugs at $0.5 \mu \mathrm{l} / \mathrm{s}$ during 10 min. to mimic a bolus injection. Then we perfused the 5FU solution during $72 \mathrm{~h}$ at $10 \mathrm{nl} / \mathrm{s}$ and $37^{\circ} \mathrm{C}$ (Fig. 7).

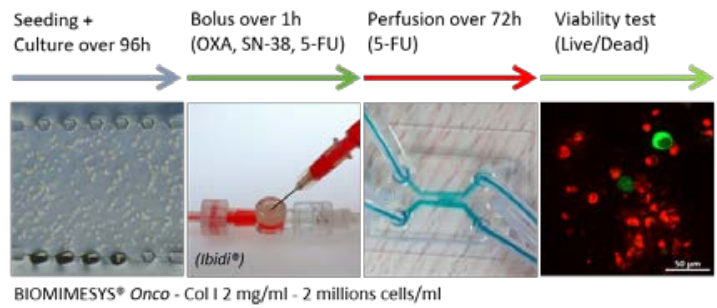

Figure 7 Description of the experimental protocol to produce IC50 curve;

The live/dead test was performed as described above for 3D static while for 3D dynamic, $300 \mu \mathrm{l}$ of solution was perfused at a flow rate of $0,5 \mu \mathrm{l} / \mathrm{sec}$ with a Legato syringe pump (KDScientific, Holliston, USA) and then incubated at room temperature for 30 minutes. a)

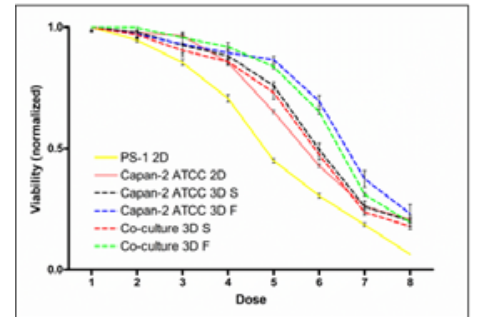

b)

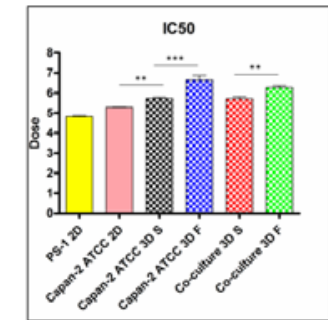

Figure 8: a) IC50 curve for FOLFIRINOX treatment; b) IC50 dose for the 6 different culture conditions. (S : static, $F$ : fluidic/dynamic)

We showed IC50 dose is culture model-dependent and that the dynamic conditions increase the chemoresistance of the tumor model (Fig. 8). With these features, our microfluidic model of PC provides more accurate conditions for screening chemotherapeutic drug activity

\section{REFERENCES}

[1] J. Kleeff, M. Korc, et al., "Pancreatic cancer”, Nat Rev Dis Primers, vol. 2, pp. 16022-1644, 2016.

[2] Q. Sun, B. Zhang, Q. Hu, Y. Qin et al., "The impact of cancerassociated fibroblasts on major hallmarks of pancreatic cancer", Theranostics, vol. 8(18), pp. 5072-5087, 2018.

[3] M.D. Nieskoski et al., "Collagen Complexity Spatially

Defines Microregions of Total Tissue Pressure in Pancreatic Cancer”, Scientific Reports, vol. 7, 10093, 2017

[4] M.A. Jacobetz, D.S. Chan, et al., "Hyaluronan impairs vascular function and drug delivery in a mouse model of pancreatic cancer”, Gut, vol. 62, pp. 112-120, 2013.

[5] M.F. Nielsen, M.B. Mortensen, S. Detlefsen, "Key players in pancreatic cancer-stroma interaction: Cancer-associated fibroblasts, endothelial and inflammatory cells", World $J$ Gastroenterol., vol. 22, pp. 2678-2700, 2016.

[6] I. Lohse, C. Lourenco, et al., "Assessment of hypoxia in the stroma of patient-derived pancreatic tumor xenografts”, Cancers, vol. 6(1), pp. 459-471, 2014.

[7] E. Tomás-Bort, M. Kieler, et al., "3D approaches to model the tumor microenvironment of pancreatic cancer”, Theranostics, vol. 10(11), pp. 5074-5089, 2020.

[8] K. Zeeberg, R.A. Cardone, M.R. Greco, et al., “Assessment of different 3D culture systems to study tumor phenotype and chemosensitivity in pancreatic ductal adenocarcinoma", International Journal of Oncology, vol. 49, pp. 243-252, 2016.

[9] C.R. Drifka, K.W. Eliceiri, et al., "A bioengineered heterotypic stroma-cancer microenvironment model to study pancreatic ductal adenocarcinoma”, Lab Chip, vol. 13: pp. 39653975, 2013.

[10] M. Beer, N. Kuppalu, M. Stefanini, H. Becker, I. Schulz, S. Manoli et al., "A novel microfluidic 3D platform for culturing pancreatic ductal adenocarcinoma cells: comparison with in vitro cultures and in vivo xenografts”, Sci Rep., vol. 7, p. 1325, 2017.

[11] D. Gioeli, C.J. Snow, et al., "Development of a multicellular pancreatic tumor microenvironment system

using patient-derived tumor cells”, Lab Chip, vol. 19, pp. 1193204, 2019.

[12] J.H. Lee, S.K. Kim, et al., "Microfluidic co-culture of pancreatic tumor spheroids with stellate cells as a novel 3D model for investigation of stroma-mediated cell motility and drug resistance”, J. Exp Clin Cancer Res., vol. 37, p. 4, 2018.

[13] H. Cho, H.-Y. Kim, J. Y. Kang, T.S. Kim, "How the capillary burst microvalve works", Journal of Colloid and Interface Science, vol. 306, pp. 379-385, 2007.

[14] C.P Huang, J. Lu et al., "Engineering microscale cellular niches for three-dimensional multicellular co-cultures”, Lab Chip, vol. 9(12), pp. 1740-1748, 2009.

[15] J.M. Rutkowski and M.A. Swartz, "A driving force for change: interstitial flow as a morphoregulator", TRENDS in Cell Biology, vol. 17(1), pp. 44-50, 2006.

[16] M. Baydoun, A. Treizebré, J. Follet, et al., “An interphase microfluidic culture system for the study of ex vivo intestinal tissue”, Micromachines, vol. 11(2), pp. 150-166, 2020.

[17] V.S. Shirure, et al., "Tumor-on-a-chip platform to investigate progression and drug sensitivity in cell lines and patient-derived organoids”, Lab Chip, vol. 18, pp. 3687-3702, 2018.

[18] F.E. Froeling, T.A. Mirza, R.M. Feakins et al., "Organotypic Culture Model of Pancreatic Cancer Demonstrates that Stromal Cells Modulate E-Cadherin, $\beta$-Catenin, and Ezrin Expression in Tumor Cells”, Am J Pathol., vol. 175(2), pp. 636-648, 2009.

[19] D. Tang, D. Wang, Z. Yuan, et al., "Persistent activation of pancreatic stellate cells creates a microenvironment favorable for the malignant behavior of pancreatic ductal adenocarcinoma”, Int. J. Cancer, vol. 132, pp. 993-1003, 2013.

[20] S.K. Begg, et al., "FOLFIRINOX Versus Gemcitabine-based Therapy for Pancreatic Ductal Adenocarcinoma: Lessons from Patient-derived Cell Lines”, 2020. 